\title{
A VECTOR MEASURE WITH NO DERIVATIVE
}

\author{
D. R. LEWIS
}

\begin{abstract}
Given a nonatomic scalar measure $\mu$, there is a vector valued, $\mu$-continuous measure of finite variation which has no derivative with respect to $\mu$, but which has the property that the closure of its range is compact and convex.
\end{abstract}

In this note we give an example of a vector measure which answers a question raised in [5]. Further, the example can be used to gain some information about certain Banach spaces with the Radon-Nikodym property. Measure theoretic terminology is that of [5]. The Banach space (under supremum norm) of all real sequences which converge to zero is written $c_{0}$. An element of $c_{0}$ will be denoted by a doubly indexed sequence $\left(a_{n, i}\right)$, with $n \geqq 1$ and $2^{n} \leqq i<2^{n+1}$.

THEOREM. Suppose that $(S, \Sigma, \mu)$ is a finite nonnegative measure space which has no atoms. There is a measure $\varphi: \Sigma \rightarrow c_{0}$ such that

(1) $\varphi$ is $\mu$-continuous and of finite variation,

(2) the closure of the range of $\varphi$ is compact and convex, and

(3) $\varphi$ has no Bochner integrable derivative with respect to $\mu$.

Proof. Since $\mu$ has no atoms we may generate a doubly indexed sequence $\left(A_{n, i}\right), n \geqq 1$ and $2^{n} \leqq i<2^{n+1}$, of measurable sets such that $\mu\left(A_{n, i}\right)=2^{-n} \mu(S)$ and $A_{n, i}$ is the disjoint union of $A_{n+1,2 i}$ and $A_{n+1,2 i+1}$. Define $\varphi: \Sigma \rightarrow c_{0}$ by $\varphi(A)=\left(\mu\left(A \cap A_{n, i}\right)\right)$. Clearly $\|\varphi(A)\| \leqq \mu(A)$ for all measurable $A$, so $\varphi$ must be $\mu$-continuous and of finite variation.

The range of $\varphi$ is contained in the set $M$ of sequences $\left(a_{n, i}\right)$ such that $0 \leqq a_{n, i} \leqq 2^{-n} \mu(S)$ and $a_{n, i}=a_{n+1,2 i}+a_{n+1,2 i+1}$ for $2^{n} \leqq i<2^{n+1}$. The sequences in $M$ converge to zero uniformly so $M$ is relatively compact, and it is clear that $M$ is closed and convex. For $\left(a_{n, i}\right) \in M$ and $\varepsilon>0$, choose $m \geqq 1$ so that $2^{-m} \mu(S)<\varepsilon$. Again using the fact that $\mu$ has no atoms there are measurable sets $B_{i} \subset A_{m, i}, 2^{m} \leqq i<2^{m+1}$, satisfying $\mu\left(B_{i}\right)=a_{m, i}$. Then $\left\|\varphi(B)-\left(a_{n, i}\right)\right\|<\varepsilon$, where $B=\bigcup_{2^{m} \leqq i<2^{m+1}} B_{i}$.

To see that $\varphi$ has no derivative with respect to $\mu$, we suppose the contrary. Let $f: S \rightarrow c_{0}$ be a derivative of $\varphi$ and write $\left(e_{n, i}\right)$ for the unit vectors

Received by the editors June 14, 1971.

AMS 1970 subject classifications. Primary 28A45, 46G10.

Key words and phrases. Vector measure, derivative of a vector measure, range of a vector measure, Radon-Nikodym property.

(c) American Mathematical Society 1972 
in $l_{1}$. For each $i \geqq 2$ and $A \in \Sigma$,

$$
\int_{A}\left\langle f(s), e_{n, i}\right\rangle \mu(d s)=\mu\left(A \cap A_{n, i}\right),
$$

so there is, for each $i$, a $\mu$-null set $C_{i} \subset S$ such that $\left\langle f(s), e_{n, i}\right\rangle=\chi_{A_{n, i}}(s)$ for $s \notin C_{i}$. Choose $t \in S \backslash \bigcup_{i} C_{i}$. By the way in which the sets $\left(A_{n, i}\right)$ were chosen $\chi_{A_{n, i}}(t)=1$ for infinitely many indices $i$, so $\lim _{i}\left\langle f(t), e_{n, i}\right\rangle \neq 0$. This is the desired contradiction.

A Banach space $E$ is said to have the Radon-Nikodym property (rn) if, given any finite measure space $(S, \Sigma, \mu)$, every $\mu$-continuous measure of finite variation $\varphi: \Sigma \rightarrow E$ has a Bochner integrable derivative with respect to $\mu$ (the classical examples are the reflexive spaces, separable duals and $\left.l_{1}(\Gamma)\right)$. Little is known about the structure of such Banach spaces. The example given above indicates that no Banach space $E$ with a subspace isomorphic to $c_{0}$ has this property. Stated affirmatively [1] this means that each weakly unconditionally Cauchy sequence in $E$ is unconditionally convergent. It is known [4] that a complemented subspace of $L_{1}(\mu)$ which has $(\mathrm{rn})$ is isomorphic to $l_{1}(\Gamma)$ for some set $\Gamma$. The example given above shows that a quotient of $C(S)$ ( $S$ compact Hausdorff) with (rn) must be reflexive [3], and that a complemented subspace of $C(S)$ with (rn) must be finite dimensional. Finally, let us note that this example, combined with the Radon-Nikodym theorem of Dunford and Pettis [2] for measures into separable duals, gives an easy proof of the classical result of Orlicz that no separable dual contains $c_{0}$.

\section{BIBLIOGRAPHY}

1. C. Bessaga and A. Pelczyński, On bases and unconditional convergence of series in Banach spaces, Studia Math. 17 (1958), 151-164. MR 22 \#5872.

2. N. Dunford and B. J. Pettis, Linear operations on summable functions, Trans. Amer. Math. Soc. 47 (1940), 323-392. MR 1, 338.

3. A. Pelczyński, Banach spaces on which every unconditionally converging operator is weakly compact, Bull. Acad. Polon. Sci. Ser. Sci. Math. Astronom. Phys. 10 (1962), 641-648. MR 26 \#6785.

4. D. R. Lewis and C. Stegall, Banach spaces whose duals are isomorphic to $l_{1}(\Gamma)$ (submitted).

5. J. J. Uhl, Jr., The range of a vector-valued measure, Proc. Amer. Math. Soc. 23 (1969), 158-163. MR 41 \#8628.

Department of Mathematics, Virginia Polytechnic Institute and State UniverSity, Blacksburg, Virginia 24061 